\title{
A review of Artificial Intelligent approaches applied to part accuracy prediction
}

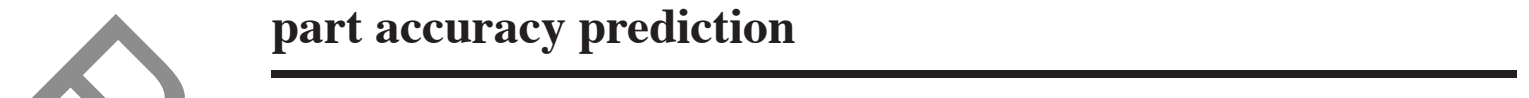

\section{J.V. Abellan-Nebot}

Department of Industrial Systems Engineering and Design, School of Technology and Experimental Sciences, Universitat Jaume I, 12071 Castellon, Spain

Fax: +34964728170Ｅ-mail: abellan@esid.uji.es

\section{Abstract}

Nowadays, despite the large volume of worldwide academic research on various aspects of metal cutting the control of workpiece precision still relies on machine-tool operator's experience and trial and error runs. In order to increase the efficiency of machining systems, many empirical models based on Artificial Intelligent (AI) approaches have been proposed in the past, where important process improvements were reported. This paper overviews the AI approaches applied in machining operations to predict part accuracy in terms of dimensional deviations and surface roughness. Successful techniques applied in this field such as Artificial Neural Networks, Fuzzy Logic, Adaptive-Network-based Fuzzy Inference Systems and Bayesian Networks are briefly reviewed and compared to facilitate its use. For each AIapproach, the most relevant research works are described and based on those works some guidelines are proposed for its implementation. In addition, advantages and drawbacks of each approach are summarised and a generic guideline for AI approaches selection is proposed.

Keywords: Artificial Intelligence, Part Accuracy, Surface Roughness, Dimensional Deviations, Artificial Neural Networks, Fuzzy Logic, Adaptive-Networkbased Fuzzy Inference Systems, Bayesian Networks, Guidelines.

Reference to this paper should be made as follows:

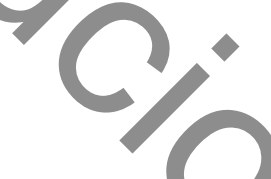

Biographical Notes: José V. Abellán-Nebot is assistant professor in the Department of Industrial Systems Engineering and Design at Jaume I University. He received the M.Sc. degree in manufacturing engineering in 2003. In 2004, he joined the Department as a research assistant. Since 2007, he lectures Computer Integrated Manufacturing, Computer Aided Manufacturing and Manufacturing Technologies. He was a visiting scholar at the Centre for Innovation in Design and Technology located at Monterrey Institute of Technology (México) in 2005 and at the Engineering Research Center for Reconfigurable Manufacturing Systems (ERC-RMS) at University of Michigan in 2007. He is author of more than fifteen conference proceedings and several research studies published at international journals. His research interests include issues related to intelligent machining systems, stream of variation in machining systems and manufacturing and collaborative engineering. 


\section{Introduction}

The control of all aspects of product quality is the ultimate technical objective of manufacturing industry. In machining operations, this mainly implies workpiece precision. Nowadays, despite the large volume of worldwide academic research on various aspects of metal cutting the control of workpiece precision in industry still relies on the machine-tool operator's experience and trial and error runs (van Luttervelt and Peng, 1999). The difficulties in realising true predictive models to estimate part accuracy in machining arise from the extreme physical phenomena inherent in the process. Machining generates a highly inhomogeneous plastic flow where local stresses generate high rates of plastic deformation that give rise to inhomogeneous thermal fields, high temperatures and pressures. This type of complex plastic flow is difficult to predict even with sophisticated numerical software (Ivester et al., 2000). These difficulties have forced model development to rely on various levels of empirical input data taken from machining tests in order to model process variables of industrial interest. Recently, the application of mathematical models based on Artificial Intelligence (AI) to learn/acquire the relationships between cutting parameters (e.g. cutting speed, feed rate, depth of cut, lubrication, etc.) and process variables (e.g. cutting force, acoustic emission, sound, vibration, spindle power, cutting temperature, etc.) have been successfully applied and it is expected that their potential use could solve many of the problems encountered in modelling with conventional techniques. Interestingly, in 1998 a CIRP keynote (van Lutervelt et al., 1998) about modelling of machining operations stated the importance of new modelling techniques based on AI. The keynote remarked that in future the application of $\mathrm{AI}$ in machining would develop machine-tools with the ability to predict the job quality and take appropriate corrective actions based on sensory feedback. However, present technology is still far away from that goal and the development of a reliable prediction strategy for predicting part accuracy is a challenge to be met on the way of developing an artificially intelligent and unmanned machine-tool (Risbood, Dixit and Sahasrabudhe, 2003).

Surprisingly, in spite of the promising field of AI applied in machining, there is no review on AI applied to part accuracy prediction, and only Benardos and Vosniakos's (2003) and Lu's (2008) research works scarcely deal with this topic. In order to overcome this limitation, this paper reviews the $\mathrm{AI}$ approaches applied in machining operations to predict part accuracy in terms of dimensional deviations and surface roughness. Successful techniques applied in this field such as Artificial Neural Networks, Fuzzy Logic, Adaptive-Networkbased Fuzzy Inference Systems and Bayesian Networks are briefly reviewed and compared to facilitate its use. For each AI approach, the most relevant research works are described and based on those works some guidelines are proposed for its implementation In addition, advantages and drawbacks of each approach are summarised and a generic guideline for AI approaches selection is proposed.

\section{Part accuracy in machining}

\subsection{Macro-geometrical part accuracy: Geometrical/Dimensional errors}

Many sources of geometrical/dimensional errors affect the final part accuracy in machining. These errors can be classified into two categories namely quasi-static errors and dynamic errors (Ramesh, Mannan and Poo, 2000). Quasi-static errors are those between the tool and the workpiece that are slowly varying with time and related to the structure

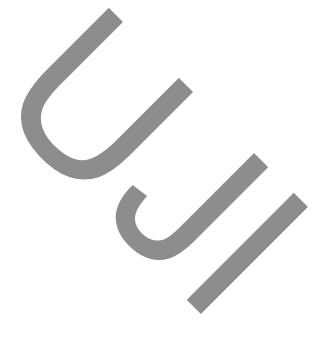


of the machine tool itself. These sources can be summed up about 70 percent of the total error of the machine-tool and they include the geometric/kinematic errors, thermal errors, cutting-force-induced errors, tool-wear-induced errors, fixturing errors, etc. (Ramesh, Mannan and Poo, 2000). On the other hand, dynamic errors are fast-changing errors caused by sources such as spindle error motion, vibrations of the machine structure and controller errors which are more dependent on the particular operating conditions of the machine. Fig. 1 shows the usual quasi-static and dynamic error sources in machining.

In order to assure minimal geometrical/dimensional deviations, these factors should be monitored to quantify the final deviation from the nominal part values. In this field most of the works conducted have been focused on analytical and mechanistic approaches (e.g. Chen (2000); Raghu and Melkote (2005); de Lacalle et al. (2004)) and a lower number of works have been carried out using empirical approaches based on AI techniques (e.g. Ouafi, Guillot and Bedrouni (2000); Risbood, Dixit and Sahasrabudhe (2003); Warnecke and Kluge (1998)).

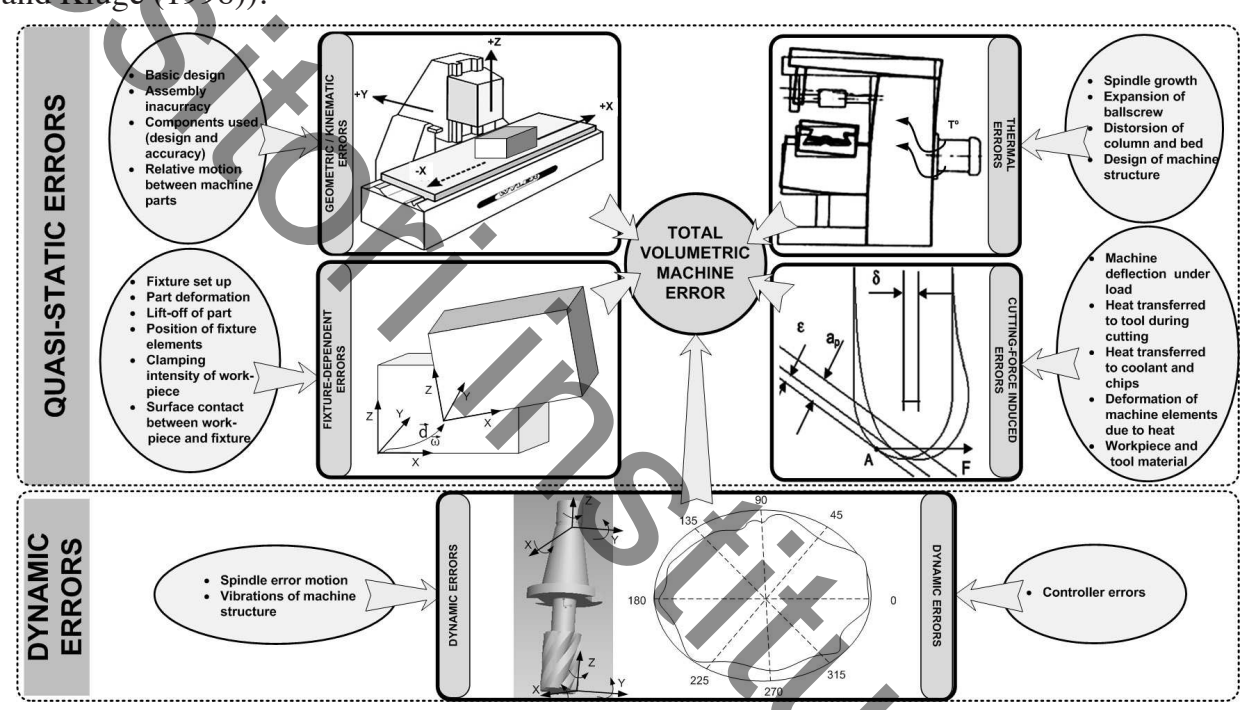

Figure 1 Error sources affecting macro-geometrical part accuracy, Quasi-static errors can be summed up about 70 percent of the total volumetric machine error.

\subsection{Micro-geometrical part accuracy: Surface roughness}

According to international standards (DIN4760, 1982), surface roughness refers to deviation from the nominal surface of the third up to sixth order. First- and second-order deviations refer to form (i.e. flatness, circularity, etc.) and to waviness respectively, and are due to machine-tool errors, deformation of the workpiece, vibration and workpiece material inhomogeneities. Third- and fourth-order deviations refer to periodic grooves, cracks and dilapidations, which are connected to the shape and condition of the cutting edges, chip formation and process kinematics. Fifth- and sixth-order deviations refer to workpiece material structure, which is connected to physical-chemical mechanisms acting on a grain and lattice scale (slip, diffusion, oxidation, residual stress, etc.).

The importance of surface roughness in machining relies on its great influence on the tribological properties, fatigue strength, corrosion resistance and aesthetic appeal of the 
product (Risbood, Dixit and Sahasrabudhe, 2003). In spite of the great efforts to predict surface roughness, the complex mechanism behind the formation of surface roughness more than 20 factors affects surface roughness generation, Fig. 2. makes unreliable to evaluate it through any analytical formulae. In order to overcome this limitation, many researches have developed predictive and empirical models based on sensor systems and AI approaches (e.g. Risbood, Dixit and Sahasrabudhe (2003); Abburi and Dixit (2006)) instead of analytical or mechanistic models (e.g. Grzesik (1996); Stephenson and Agapiou (1997)).

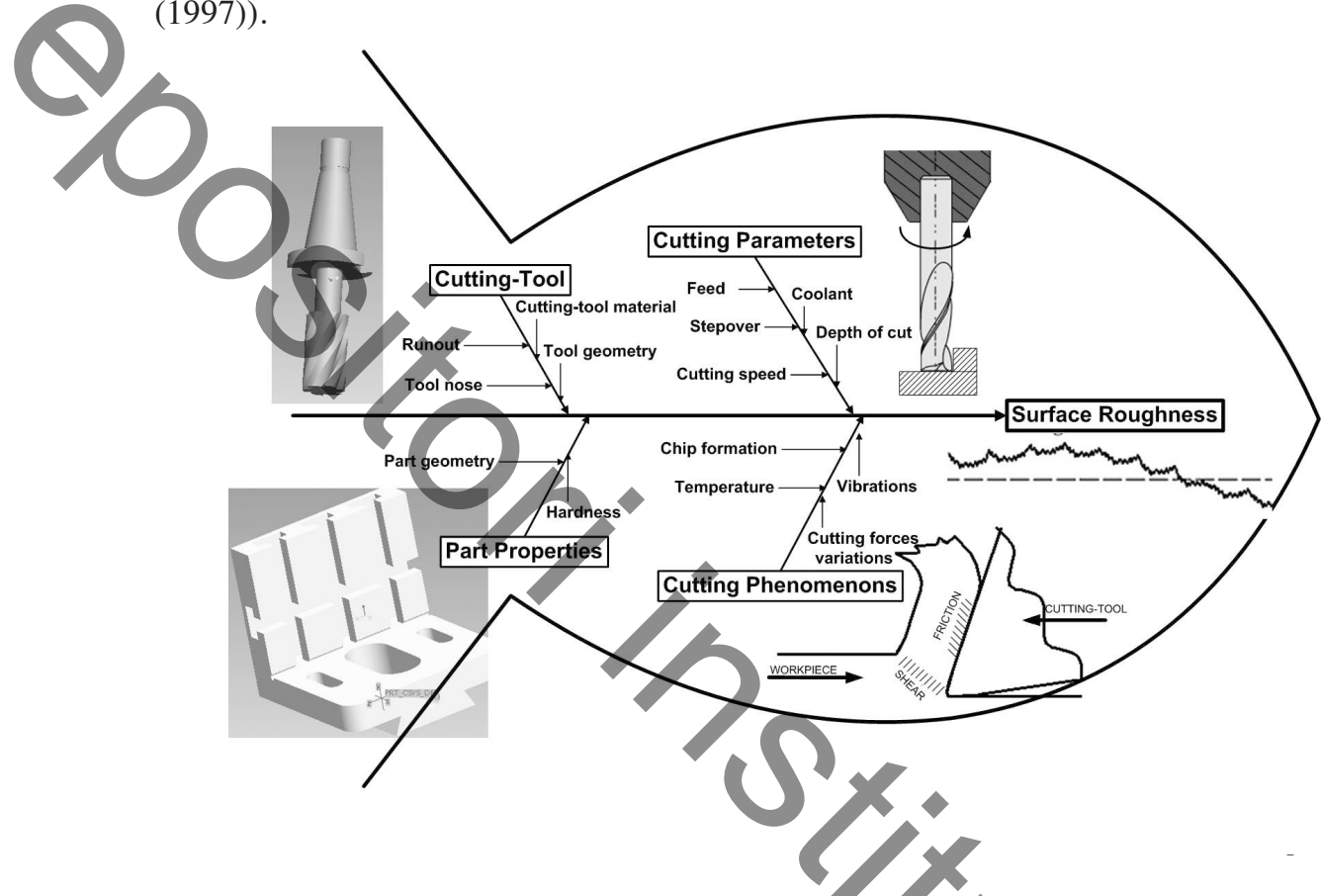

Figure 2 Factors affecting surface roughness generation -adapted from Benardos and Vosniakos's (2003) research work-.

\section{AI approaches for part accuracy prediction}

Many different AI approaches have been successfully applied for part accuracy prediction in the past. Among them, four AI approaches represent more than the $90 \%$ of the research works reported in the literature (see Fig. 3). These approaches are ArtificialNeural Networks, Fuzzy Logic, Neuro-Fuzzy systems, and Bayesian networks, and they will be briefly reviewed in the next subsections, reporting the recent research works in this field. Special interest is paid on Artificial Neural Networks, where the Multi-Layer Perceptron Neural Network (MLP NN) has been applied in more than half of the references related to part accuracy prediction. Recently, other AI techniques are gaining popularity such as evolutionary programming methods (Colak, Kurbanoglu and Kayacan, 2007) and Polynomial Networks (Chang et al., 2006) but their analysis are out of the scope of this paper.

The literature review distinguishes the AI application according to micro- and macrogeometrical predictions. Micro-geometrical estimation should deal with an important stochastic behaviour and non-linear relationships between many different cutting parameters and 


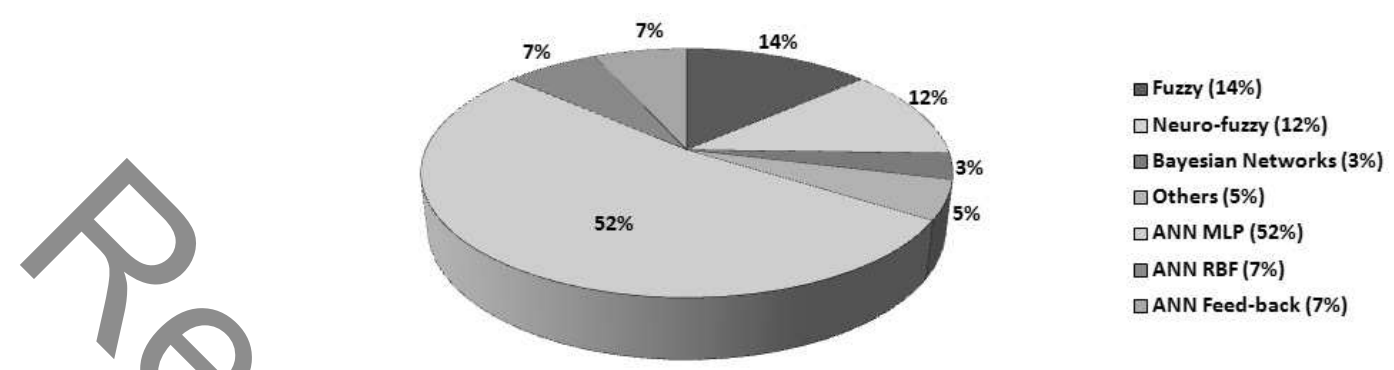

Figure 3 Frequency of usage of Artificial Intelligence approaches in part accuracy prediction. References from ISI Web of Knowledge (2003-2008)

process variables, specially in milling operations, which means that many different AI techniques could be properly applied. However, macro-geometrical estimations tend to be more heuristic and they are mostly restricted to the application of ANN approaches. For these estimations, knowledge extraction methods are less important since the main goal is to adjust non-linear functions according to clearly defined variables such as temperatures (thermal expansion) or forces (deflections).

\subsection{ANN approach}

\subsubsection{Introduction}

An Artificial Neural Network (ANN) is essentially a mathematical model that mimics the human reasoning and neurobiology. This powerfulmodelling technique is called as an "universal function approximator" due to its capacity to adjust any function. A neural network has a parallel-distributed architecture with a large number of neurons and connections which can learn non-linear relationships between variables from experimental data. ANNs are mostly used for pattern recognition, classification and systems modelling with applications ranging from simple signal processing to medical diagnosis (Kasabov, 1996).

The classification of the ANN architectures are defined by the arrangement of the interneuron connections and the nature of the connections. Basically, feed-forward and feedback networks can be distinguished. Feed-forward networks are static in the sense that they produce only one set of output values rather than a sequence of values from a given input. Popular feed-forward neural networks are Multi-Layer Perceptron (MLP) and Radial Basis Function nets (RBF). Unlike feed-forward networks, recurrent or feed-back networks are dynamic systems. When a new input pattern is presented, the neuron outputs are computed. Because of the feed-back paths, the inputs to each neuron are then modified, which leads the network to enter a new state (Jain, Mao and Mohiuddin, 1996). Popular recurrent networks are Kohonen's Self-Organising Maps (SOM), Hopfield networks and ART models.

An ANN model learns/acquires the relationship between variables through a learning process which can be viewed as a problem of updating network architecture and connection weights so that a network can efficiently perform a specific task. The network usually must learn the connection weights from available training patterns. Three main learning paradigms are distinguished: supervised, where training examples comprise input vectors $\mathbf{x}$ and the desired output vectors $\mathbf{y}$; unsupervised, where only input vectors $\mathbf{x}$ are supplied and

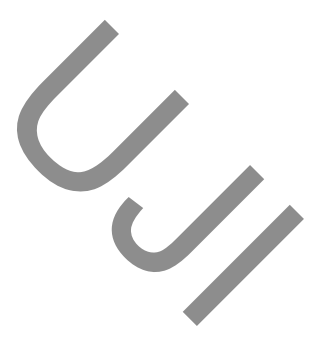


the neural network learns some internal features from the whole dataset; and hybrid, where it is combined the supervised and unsupervised learning.

An example of a generic MLP NN is shown in Fig. 4 together with two ANN models developed by Raksiri and Parnichkun (2004) and Benardos and Vosniakos (2002) for dimensional deviation and surface roughness prediction respectively.
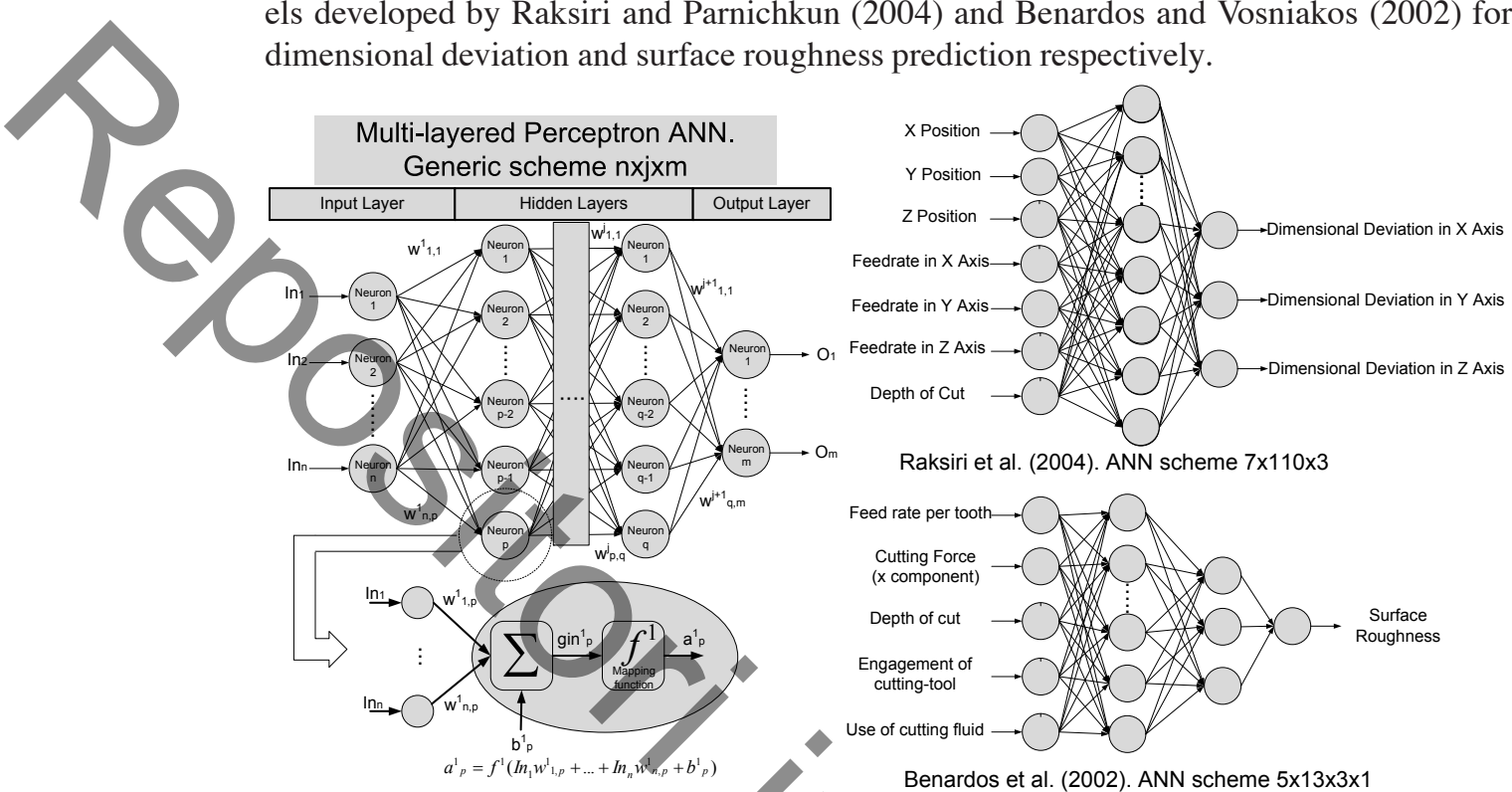

Figure 4 Generic scheme of a multi-layer perceptron artificial neural network with $n$ inputs, $j$ hidden layers and $m$ outputs. Examples reported by Raksiri et al. (2004) and Benardos et al. (2002) for dimensional deviation and surface roughness prediction respectively.

\subsubsection{ANN Models applied to part accuracy prediction}

Macro-geometrical errors in machining have been commonly estimated in the past by ANN networks due to their ability to capture nonlinear relationship between experimental variables.

In turning operations, special attention was paid to cutting-force induced deflections. Azouzi and Guillot (1997) presented a sensor fusion system based on a MLP NN to predict dimensional deviations in turning operations by monitoring vibrations, acoustic emission, and cutting forces. The ANN model prediction was assessed with an error varying from 2 and $20 \mu \mathrm{m}$ under different process conditions. The ANN model used only the cutting forces measurements as input, discarding the acoustic emission and vibration measurements.Warnecke and Kluge (1998) included the cutting-tool wear as additional input to keep under control the dimensional tolerances in a turning process. The research work combined an analytical model to predict the deformation of the workpiece and machine, and an empirical model based on ANN to predict the flank wear and the dimensional deviations due to tool wear. The ANN model for cutting-tool wear prediction used as inputs the cutting parameters and the acoustic emission measurements from the cutting-tool. The width of the wear mark was predicted with a precision of $\pm 20 \mu \mathrm{m}$ and consequently, the displacement of the cutting edge caused by tool wear and dimensional deviations was forecasted with a

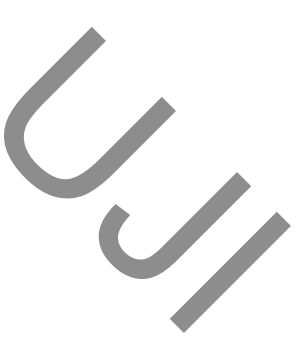


precision of $\pm 2 \mu \mathrm{m}$. Ouafi, Guillot and Bedrouni (2000) expanded the previous works by including the three main sources of dimensional deviations in turning operations namely geometric, thermal and dynamic errors. In order to monitor and estimate these errors, they presented a multilayer feed-forward neural network which fused sensor information from a dynamometer and several thermistors. The neurocompensation approach improved the machine accuracy by reducing the maximum error without compensation from $70 \mu \mathrm{m}$ to less than $4 \mu m$.

In milling operations the dimensional deviations tend to be greater than turning operations due to the higher complexity of cutting tool movements. Raksiri and Parnichkun (2004) presented an off-line error compensation model considering geometric and cutting force induced errors in a 3-axis CNC milling machine for slot cutting operations with flat end mills. In this work, three positional errors, 9 angular errors, 6 straightness errors and 3 squareness errors were measured by laser interferometer in order to estimate the geometric machine-tool error. Function approximation by back-propagation neural network was used to approximate the geometric error model, where the positions $x, y$ and $z$ of the measuring point were the input of the network and the geometric error in $x, y$ and $z$ axis were the output of the network. The investigation of cutting force induced error was carried out in $x$ forward and backward directions and $y$ forward and backward directions using a cutting force sensor and a camera. The experimental results showed that the machine accuracy was improved significantly, decreasing the machining errors from 200-300 $\mu \mathrm{m}$ to less than 40 $\mu m$.

The limitations of traditional feed-forward NNs such as their fixed architecture and the difficulties of these feed-forward models to deal with time have led to the application of other connectionist models (Bernauer and Demmou, 1993). Mize and Ziegert (2000) developed a neural network ART to predict and compensate the tool point errors of a 3-axis machining centre using discrete temperature readings from the machine's structure as inputs. A combination of kinematic error modelling, curve fitting, and neural networks were used to maintain the machine's three-dimensional accuracy within $67.4 \mu \mathrm{m}$, regardless of the thermal state. Similarly, a dynamic feed-forward NN was developed by Chang et al. (2006) to predict thermal deformation in machine tools. The dynamic ANN model showed a more accurate dimensional deviation prediction than a conventional neural network, decreasing the maximum prediction error from $5 \mu m$ to $3 \mu m$.

\section{- Micro-geometrical part accuracy}

Surface roughness has been commonly predicted by empirical models using AI techniques, specially ANNs. Low cost and non-intrusive sensors such as accelerometers and acoustic emission sensors have been intensively studied in order to let surface roughness prediction systems to be implemented in industry. Lee and Chen (2003) developed an on-line surface recognition system based on neural networks and vibration measurements in a turning operation. The MLP neural network with two hidden layers predicted the surface roughness with high accuracy (90\%). Similarly, Kohli and Dixit (2005) developed MLP NN models for surface roughness prediction in dry and wet turning operations for high speed steel tools and carbide tools using as feed-back the radial vibration of the tool holder. The networks were trained by the back-propagation algorithm and the learning rate, the number of neurons in the hidden layer, and the training and testing dataset size were found automatically in an adaptive manner. In their investigation, special attention was paid to the data filtration scheme required to build ANN models reliable and accurate. Azouzi and Guillot (1997) presented a sensor fusion system based on a MLP NN where vibrations, acoustic emission, 
forces and deflexion measurements were analysed and included in the model to predict both surface roughness and dimensional deviations. The ANN model for surface roughness prediction was assessed with an error varying from 2 to $25 \%$ under different process conditions.

Pal and Chakraborty (2005) presented a surface roughness model based on ANN for a milling operation where cutting force measurements were included. The inputs of the neural network model were the cutting speed, feed rate and depth of cut as process parametric conditions, and the feed and cutting forces as the measured parameters. In the network, the number of hidden layer, number of nodes in the hidden layer, learning rate, and momentum coefficient were decided by trial and error. The training procedure was conducted using 20 data sets from 27 combinations of experiments on mild steel work-pieces using high speed steels as cutting tools. However, the work seems not to clarify the 20 data sets composition applied. In fact, the optimal neural network structure selected was a 5-5-1 network which requires at least 30 free parameters to be learnt (if biases are not considered), so training data should be composed of more than 30 data sets in order to avoid mathematical indetermination in the neural network training process. In addition, different initialisation weights during training were not provided, which could report if part of the parameters learnt depend on the initialisation values due to the lack of data training samples. The final surface roughness model prediction was within an accuracy of $\pm 5 \%$.

Minimal experimental data for modelling purposes was considered by Benardos and Vosniakos (2002) as an important issue to facilitate the implementation of ANN models in industry. They proposed the application of Taguchi design of experiments, and validated an ANN model in face milling operations with aluminium alloys to predict surface roughness. The process factors considered as ANN inputs were: feed rate per tooth, depth of cut, engagement of the cutting tool, use of cutting fluid and the component of the cutting force along the direction of the feed. The results of this study (mean squared error less than $1.86 \%$ ) confirmed the high accuracy of ANNs for modelling machining operations with considerably few experimental data. Benardos also presented a trial an error procedure to obtain an optimal number of layers and neurons avoiding overfitting through the early stopping methodology.

One of the most important limitations of ANN models is the selection of the proper ANN structure which leads to the best ANN model. Most of the investigations presented in the literature applied a trial and error procedure as it is presented in Benardos and Vosniakos's (2002) research work. However, other researchers propose the development of MLP $\mathrm{NN}$ models using Bayesian regularisation with Levenberg-Marquardt training algorithm to partly overcome this problem. In Ozel and Karpat's (2005) research work, the Bayesian regularisation method was applied to determine the optimum number of neurons in the hidden layer of ANN models in order to predict the surface roughness and tool wear in hard turning operations using Cubic Boron Nitride (CBN) tools.

A part from MLP NN, other types of ANN models have been applied for surface roughness prediction with less efforts. For example, Sonar, Dixit and Ojha (2006) developed a RBF NN for predicting the surface roughness in a turning process, and they compared its performance with a MLP NN. Although the RBF network was simpler and required a less computational time, its performance was slightly inferior than the MLP NN.

Another important limitation of ANN models which prevents their application in industry is related to their performance when the machining process varies. Risbood, Dixit and Sahasrabudhe (2003) trained different MLP network topologies in a turning process for both surface roughness and dimension deviation prediction. He incorporated into the

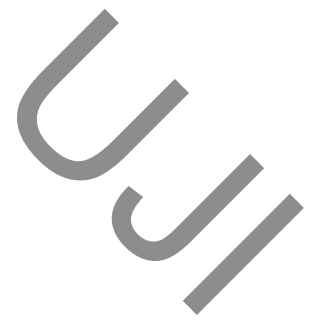


network cutting forces measurements and radial vibrations although the cutting forces were finally discarded due to their insignificance in the prediction accuracy. The networks developed were trained by back-propagation algorithm, with sigmoid and pure linear functions as transfer and output functions respectively. The best network after testing different neuron combinations was a MLP with three neurons in a hidden layer, with a maximum error in the surface roughness prediction of $18.21 \%$. A similar ANN structure was developed for dimensional deviation prediction, and the model was validated with a prediction error less than $40 \mu \mathrm{m}$. Interestingly, this work reported that a change on the cutting-tool geometry required few additional experiments to adapt the ANN model to the new machining process. In addition, other changes were studied such as a change from dry to wet turning and a change from HSS to carbide tools. For these cases, the ANN model had to be training again in order to be able to predict the surface roughness of these new machining processes, showing the limitations of these models for industrial applications. Recently, Abellan-Nebot et al. (2008) studied how the prediction error of a surface roughness ANN model increases when cutting-tool changes occur. It was tested that for slight cutting-tool changes the ANN model was able to be re-trained by few experimental data in order to increase the prediction reliability. However, severe cutting-tool changes where other factors such as run-out effects may show up require to develop an ANN model from the scratch to keep the prediction accuracy with a reasonable error. Zhong, Khoo and Han (2006) also tested how the surface roughness prediction error by an ANN model varies when the machining operation is conducted in different machine-tools. The experimentation showed an increase of the average prediction error from $15.2 \%$ to $24.4 \%$ when the model was applied in a lathe different from the one where the model was trained.

Finally, other interesting research works compare the effectiveness of ANN models for part accuracy prediction versus other modelling techniques. Tsai, Chen and Lou (1999) developed an in-process based surface recognition system to predict the surface roughness of machined parts in the end milling process. The parameters included in the prediction model were the spindle speed, feed rate, depth of cut and the vibration average per revolution measured by an accelerometer. Different models were developed using both MLP NN and statistical multiple regressions for comparison purposes. The experimental results concluded that the ANN models behaved better under all tested situations. Other interesting comparisons were conducted by $\mathrm{Li}$, Guan and $\mathrm{Li}$ (2004), where the dimensional deviation in end milling operations was predicted by an RBF NN, an hybrid RBF NN and an ANFIS model. The results reported a prediction error of $15 \%, 25 \%$ and $5 \%$ when using the RBF, the hybrid RBF and the ANFIS model respectively. ANN models were also compared with Bayesian networks and statistical multiple regressions by Abellan-Nebot et al. (2006b) and Correa, Bielza and Pamies-Teixeira (2008b). The conclusions of these works seem contradictory since Abellan-Nebot et al. (2006b) reported a higher aceuracy of ANN models versus BN since they used both methodologies as function approximators. However, Correa, Bielza and Pamies-Teixeira (2008b) showed that BNs achieve the best results from the point of view of classifier goodness applied to the problem of quality prediction in high-speed milling processes.

\subsubsection{Guidelines for ANN Modelling}

According to previous research studies on ANN models for part accuracy prediction, some important guidelines can be extracted when applying neural networks:

- ANN Type: 
Surface roughness and dimensional deviation prediction are commonly considered as a function approximation problem. This problem is successfully dealt with feedforward neural networks such as MLP and RBF, specially by the former which is the most common ANN type applied for part accuracy prediction (see Fig. 3). There are special cases where RBF networks could be recommended versus MLP since these networks present a fast training convergence, better generalisation and no local minima problems (Kasabov, 1996). However, finding the appropriate number of hidden nodes in RBF networks are specially complex. In addition, too many or too few hidden nodes will prevent RBF networks from properly approximating the data. The hidden nodes in RBF tend to have many times more neurons than a comparable feedforward network with sigmoid mapping functions in the hidden layer. As a result, the larger the input space (in terms of number of inputs, and the ranges those inputs vary over) the more radial basis neurons required. A comparison between MLP NN and RBF NN applied to surface roughness prediction is shown in Sonar, Dixit and Ojha's (2006) research work. In this work, it was observed that the performance of the RBENN was slightly inferior compared to MLP NN, although the RBF NN training procedure was simpler and required less computational time.

- ANN Structure:

There is no optimal ANN structure of MLP networks for surface roughness and/or dimensional deviation prediction and the optimal structure is considered as a trial and error problem. In spite of the considerable research done to investigate whether additional hidden layers increase the learning capacity or speed of the network, there is no convincing theoretical argument in favour of additional hidden layers, and no example has been given of a mapping problem that can be solved with multiple hidden layers but not with just a single hidden layer (Kasabov, 1996). Furthermore, White's theorem states that one layer with non-linear functional relationship is sufficient to map any non-linear functional relationship with a reasonable level of accuracy (Eberhart, Simpson and Dobbins, 1996).

- Number of hidden neurons in hidden layer:

The number of neurons in the hidden layer is the most critical parameter affecting the accuracy of the network. Too few neurons can lead to underfitting whereas too many neurons can contribute to overfitting, in which all training points are well fitted but the fitting curve oscillates wildly between these points. So there exists an optimum number of neurons in the hidden layer, which has less training error, as well as acceptable value of testing error. Ozel and Karpat (2005) applied a systematical approach for choosing the number of hidden neurons by using the output parameters of Bayesian regularisation algorithm. By applying this algorithm it is assumed that the resultant neural network has enough number of parameters to represent the training set. On the other hand, ANN theory also recommends to remove hidden units whose weights change very little from their starting values. These nodes hardly participate in the learning process, and they can be removed to simplify the network (Freeman and Skapura, 1991). In spite of these strategies for choosing the number of hidden neurons, the number of neurons of ANN models related to part accuracy have been commonly obtained by trial and error procedures using ANN structure combinations of one, two and three hidden layers (e.g. Tsai, Chen and Lou (1999); Pal and Chakraborty (2005)). 
- Mapping/training functions:

Traditionally, two mapping functions have been widely applied in MLP with backpropagation: logsig and tansig. Different authors concluded that both logsig and tansig functions produce almost the same performance and it is not a critical parameter for modelling part accuracy (Kohli and Dixit, 2005; Zhong, Khoo and Han, 2006). However, other authors consider the mapping function selection an important factor for ANN models. For example, Oktem, Erzurumlu and Erzincanli (2006) tested in their work that tangent hyperbolic function leads to a minimum training error for the prediction of surface roughness.

Training parameters:

Training Algorithm:

ANN networks for part accuracy are reduced in size due to the limitations on experimental data. Levenberg-Marquardt training algorithm (a variation of the classic back-propagation algorithm) is recommended for those applications where small and medium size networks are applied instead of gradient descent algorithm or momentum variation. Through this training algorithm, ANN part accuracy models can be trained much faster (Benardos and Vosniakos, 2002; Ozel and Karpat, 2005; Demuth, Beale and Hagan, 2008) and their generalisation capability can be improved applying the Bayesian regularisation algorithm during training.

- Learning rate:

A smaller value of the learning parameter $\eta$ will cause slow learning, however it can locate deep minima. A larger value of $\eta$ will speed up the convergence but the network may become unstable (Dixit and Chandra, 2003). A number of researchers have attempted to change $n$ dynamically by taking the feed-back of convergence behaviour (Haykins 1994). However, the implementation of this makes the code complicated, and in most cases the changing learning rate dynamically did not have a significant effect on convergence speed (Dixit and Chandra, 2003). Therefore, $\eta$ is usually found by hit and trial and the same value of $\eta$ is used for all cases.

- Initialisation:

Due to the outcome of the training greatly depends on the initialisation of the weights, this initialisation should be done randomly. In order to compare different ANN performances, each ANN has to be tested with different initialisations and an average performance has to be evaluated for comparison purposes. The Nguyen-Widrow technique is recommended for weights initialisations (Benardos and Vosniakos, 2002). In addition, advanced methods for weights initialisations have been also applied for part accuracy prediction (Jesuthanam, Kumanan and Asokan, 2007).

\section{- Generalisation}

Early stopping and Bayesian regularisation can ensure network generalisation when they are applied properly. With early stopping, the choice of the validation set is very important as it should be representative of all points in the training set. With Bayesian regularisation, it is important to train the network until it reaches convergence. The sum-squared error, the sum-squared weights, and the effective number of parameters

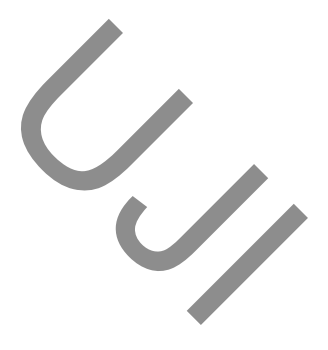


should reach constant values when the network has converged. Using both early stopping and Bayesian regularisation, it is recommended to train the network starting from several different initial conditions in order to verify the network robustness. For function approximation networks with a small data set, Bayesian regularisation provides better generalisation performance than early stopping (Demuth, Beale and Hagan, 2008). In order to improve generalisation, both early stopping and Bayesian regularisation methods are recommended and both of them are usually applied in ANN models for part accuracy prediction (e.g. Benardos and Vosniakos (2002); Ozel and Karpat (2005)).

Using a ANN:

When using the ANN predictions for part accuracy prediction, it is important to take into account not only the predicted value but also the range within the real value can lie. A methodology to predict the lower and upper bound of any non-linear function has been proposed by Ishibuchi and Tanaka (1991), which requires a simple modification of the baek-propagation algorithm. This methodology was applied by Kohli and Dixit (2005) and Sonar, Dixit and Ojha (2006) to predict the lower and upper bound of a surface roughness prediction with MLP NN and RBF nets respectively.

- Data sets: For industrial purposes, experimental sample size is restricted so special attention must be paid in avoíding noisy data and selecting independent data samples. To avoid faulty data, proper filtration schemes should be applied (e.g. in Kohli and Dixit (2005)). When sample size is reduced, three data sets should be defined after experimental acquisition. It is common to allocate 40 percent of experimental samples as training data samples, 30 percent as validating data and 30 percent as testing data. Training data are used to learn the proper neural weights; validation data to decide when to stop training and assure generalisation capability; and testing data to measure the expected performance of the network when it is put into service. In addition, the sample size for training should avoid mathematical indetermination. For this purpose, the training sample size should be reasonably higher than the number of free parameters to be learnt in the network structure. The number of ANN free parameters depends on the complexity of the network and it is calculated as shown in the equation below:

Param $_{\text {free }}=$

$$
=(\text { in }+1) \times n_{h d_{1}}+(\text { out }) \times\left(n_{h d_{N}}+1\right)+\sum_{i=2}^{N}\left(\left(n_{h d_{i-\gamma}}+1\right) \times n_{h d_{i}}\right)
$$

where $i n$ is the number of inputs; $n_{h d_{i}}$ is the number of neurons at the $i$ th layer; out is the number of outputs; and $N$ is the number of layers in the neural network.

\subsection{Fuzzy Logic}

\subsubsection{Introduction}

One way to represent inexact data and knowledge, closer to human like thinking, is to use fuzzy rules instead of exact rules when representing knowledge. Fuzzy systems are rulebased expert systems based on fuzzy rules and fuzzy inference. Fuzzy rules represent in a 
straightforward way "commonsense" knowledge and skills, or knowledge that is subjective, ambiguous, vague, or contradictory (Jain and Martin, 1998). A fuzzy inference system is composed of five functional blocks: a fuzzification interface which transforms the crisp inputs into fuzzy inputs; a rule base containing a number of fuzzy if-then rules; a database which defines the membership functions of the fuzzy sets used in the fuzzy rules; a decisionmaking unit which performs the inference; and a defuzzification interface which transform the fuzzy output into a crisp output. Usually, the rule base and the database blocks are jointly referred to as the knowledge base.

Fuzzy logic starts with the concept of a fuzzy set. A fuzzy set is a set without a crisp, chearly defined boundary. It can contain elements with only a partial degree of membership. A fuzzy set is an extension of a classical set. If $X$ is the universe of discourse and its elements are denoted by $x$, then a fuzzy set $\mathrm{A}$ in $X$ is defined as a set of ordered pairs $A=\left\{x, \mu_{A}(x) \mid x \in X\right\}$, where $\mu_{A}(x)$ is called the membership function of $x$ in A and means the degree that $x$ belongs to $A$. A membership function (MF) is a curve that defines how each point in the input space (universe of discourse) is mapped to a membership value (or degree of membership) between 0 and 1 . Some common membership functions are gaussian distribution functions, sigmoid curve and quadratic and cubic polynomial curves.

Fuzzy if-then rules or fuzzy conditional statements are expressions of the form "IF $x$ is $A$ THEN $y$ is $B$ ", where $A$ and $B$ are linguistic values defined by fuzzy sets on the ranges (universes of discourse) $X$ and $Y$, respectively. The if-part of the rule " $x$ is $A$ " is called the antecedent or premise, while the then-part of the rule " $y$ is $B$ " is called the consequent or conclusion. Interpreting an if-then rule involves distinct parts: first evaluating the antecedent and second applying that result to the consequent which specifies a fuzzy set be assigned to the output.

An example of fuzzy systems to predict surface roughness is shown in Fig. 5. This knowledge-based system was developed by Abburi and Dixit (2006) and it considers the cutting speed, feed rate, vibrations and depth of cut as the input variables. The fuzzy if-then rules shown were extracted from experimental data and they were checked by an expert machinist. As it is shown, to predict the surface roughness the inputs are firstly converted into fuzzy inputs and then, according to the fuzzy rules, the fuzzy inference is carried out. The final defuzzyfication method converts the surface roughness obtained by the fuzzy reasoning into a crisp value.
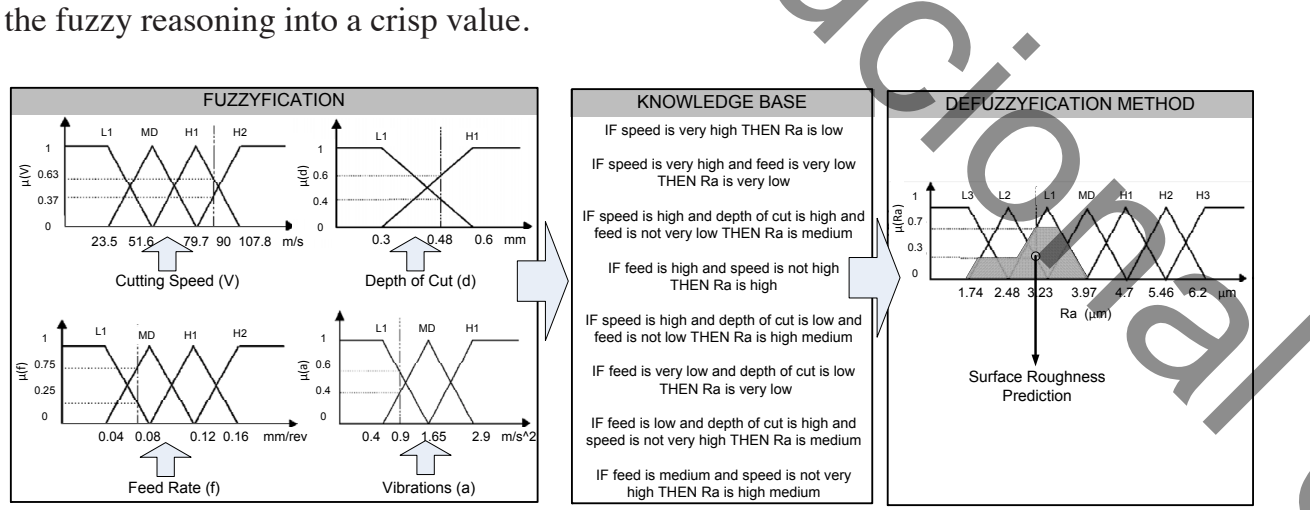

Figure 5 Fuzzy System developed by Abburi and Dixit (2006) applied to surface roughness prediction. The main parts of a fuzzy system are presented: Fuzzyfication, Knowledge Base (fuzzy reasoning), Defuzzyfication 


\subsubsection{Fuzzy Logic Models applied to part accuracy prediction}

Lou and Chen (1999) developed a surface roughness recognition system to predict surface roughness in-process. Their system was applied in end-milling operations and the input variables included were the spindle speed, feed rate, depth of cut and vibration measurements. Triangular membership functions were applied and a total of 79 fuzzy rules were extracted directly from experimental data using a product space clustering. The system was validated machining 6061 aluminium blocks with high speed steel tools and the fuzzy model was capable of predicting the surface roughness with more than $96 \%$ accuracy.

Chen and Savage (2001) expanded Lou's work by adding a new set of parameters including tool diameter and workpiece material. They also added a methodology to resolve conflicting rules after extracting them from experimental data. The system demonstrated a $90 \%$ accuracy of prediction average testing various tool diameter, tool material, and workpiece material combinations. In spite of the accuracy decrease, the Chen and Savage's (2001) research work shows the reliability of fuzzy models for surface roughness prediction in wide domains.

Iqbal et al. (2007) presented a fuzzy expert system for optimising parameters and predicting performance measures in a hard-milling process. The expert system was based on two modules, namely optimisation module and prediction module. The optimisation module provided the optimal selection of milling parameters, while the prediction module provided the prediction of performance measures such as cutting forces, tool life and surface roughness. After conducting a fractional design of experiments, the experimental data were converted to useful information using ANOVA and numeric optimisation, and this information was used to develop the knowledge-base in form of fuzzy if-then rules.

Similar works were developed for turning operations. Abburi and Dixit (2006) applied a knowledge-based system for the prediction of surface roughness in turning process with high speed steels and TiN-coated carbide tools, and rolled steel bars containing about $0.35 \%$ carbon. Instead of using the experimental data to extract the fuzzy if-then rules, a neural network which was previously trained with the experimental data was used to provide the inputs for the rule generation module. For each input-ouput neural network data set, a fuzzy rule was generated, where each variable was assigned to a fuzzy set in which it had the maximum membership grade. Due to a large number of rules prevent the operators understand the process, the bank of rules generated was reduced to a smaller set of rules by using Boolean operations. In addition, an expert machinist interacted to eliminate any incoherent rule. The system was proved to be slightly inferior to a neural network model trained in a previous research study. Most of the prediction errors in the validation data set were tested to be within $\pm 20 \%$ accuracy. However, the authors remarked the important benefits of the fuzzy system due to its extrapolation capability and its applicability for process planning (i.e, capability to select the process parameters in order to assure a desired surface roughness).

Kirby and Chen (2007) increased the accuracy of a fuzzy system for surface roughness prediction purposes by including vibrations into the model. They focused their investigation on turning operations with diamond-shape carbide tool inserts and 6061-T6511 aluminium alloy workpieces. The surface roughness prediction obtained by this fuzzy model was more accurate than previous ones, with an average accuracy of $95 \%$.

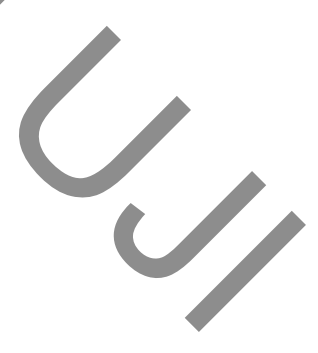




\subsubsection{Guidelines for Fuzzy logic Modelling}

The if-then rules in the fuzzy inference systems are defined by the expert machinist in order to incorporate expert knowledge into the model. However, sometimes additional rules have to be incorporated in order to define completely the fuzzy system. For this purpose, the fuzzy rules can be extracted from experimental data as it is reported in different works (Abburi and Dixit (2006), Kirby and Chen (2007), Chen and Savage (2001)). The procedure consists of generating fuzzy rules from given experimental data, assigning the value of each input as the maximum membership grade. As many rules extracted are contradictory with others, different strategies to resolve conflicting rules have to be applied, as it is reported in Kirby and Chen's (2007) research work. In addition, the extracted rules have to be reviewed by the expert machinist to discard any incoherent rule and increase the understanding of the process. The number of rules learnt has to be reasonable, since a high number of rules difficulties the understanding of the process, which is the main objective of fuzzy modelling.

The number of membership functions to define each input is closed related to the number of possible rules extracted. In general, a high number of fuzzy sets representing the input/output variables improves the resolution and the accuracy of the predictions but increases the number of rules and the complexity of the model (Abburi and Dixit, 2006). Most of the research works related to part accuracy prediction in the literature present a number of membership functions around 2-7.

There are many different membership functions to define the fuzzy sets, however it seems that there is no significative performance improvement according to membership selection. According to the literature review, most of the fuzzy systems reported applied triangular membership functions for part accuracy prediction (e.g. Abburi and Dixit (2006), Chen and Savage (2001)).

\subsection{Neuro-fuzzy Systems}

\subsubsection{Introduction}

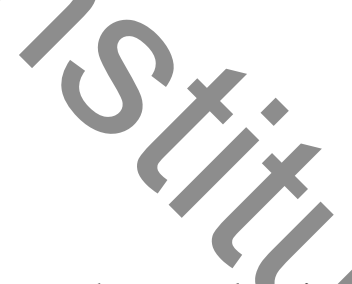

A neuro-fuzzy system is a fuzzy system that uses a learning algorithm derived from neural network theory to determine its parameters (fuzzy sets and fuzzy rules) by processing data samples. Therefore, neuro-fuzzy systems are the result of hybridising neural networks and fuzzy logic. The learning ability of neural networks let adjust the expert knowledge and generate fuzzy rules and membership functions automatically. A neuro-fuzzy system can be viewed as a 3-layer feed-forward neural network. The first layer represents input variables, the hidden layer represents fuzzy rules and the third layer represents output yariables. Fuzzy sets are encoded as fuzzy connection weights. Sometimes a 5-layer architecture is used, where the fuzzy sets are represented in the units of the second and fourth layer. A neuro-fuzzy system can be directly created from the scratch or it can be initialised with prior knowledge in form of fuzzy rules. Therefore, neuro-fuzzy is considered a technique to derive a fuzzy system from data or to enhance it by learning from examples. The most common neuro-fuzzy system applied in part accuracy prediction is a neuro-fuzzy system with a 5-layer architecture namely Adaptive-Network-based Fuzzy Inference System (ANFIS).

An example of a neuro-fuzzy system to predict surface roughness is shown in Fig. 6 , This ANFIS model was developed by Abellan-Nebot (2009) and it was applied for cutting parameter optimisation in high quality machining operations. The cutting speed and

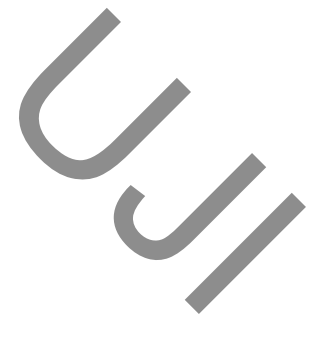


the feed rate were considered the inputs of the system, and the initial membership functions were modified after training, extracting the fuzzy rules which explained the surface roughness generation.

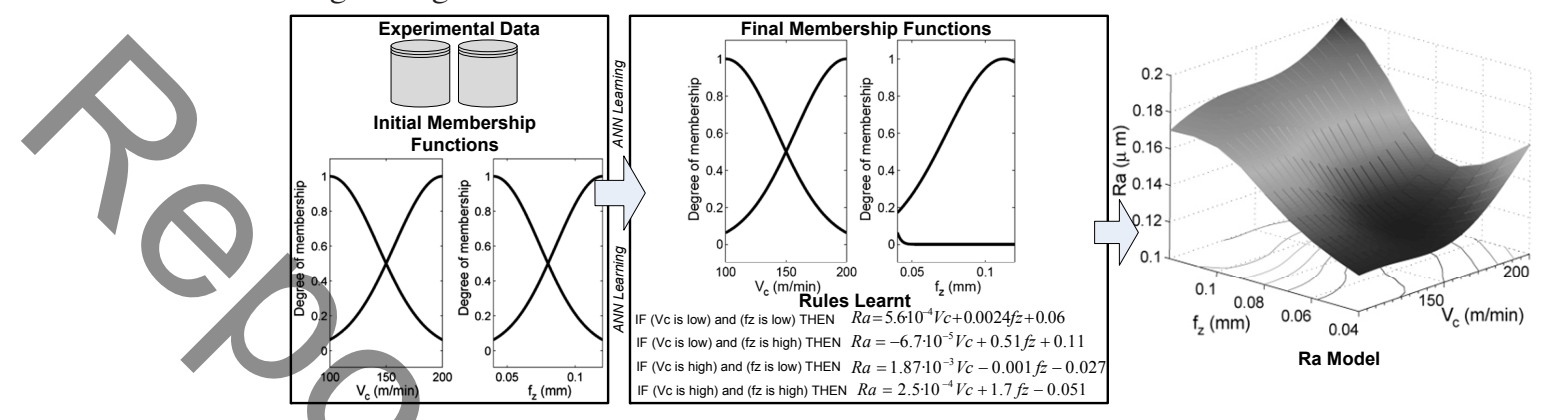

Figure 6 ANFIS model developed by Abellan-Nebot (2009) applied to surface roughness prediction and cutting parameters optimisation. The initial membership functions are modified after training, extracting the fuzzy rules which explain the surface roughness generation.

\subsubsection{Neuro-Fuzzy Logic Models applied to part accuracy prediction}

The hybridisation of neural networks and fuzzy systems have been recently applied for surface roughness prediction. Dweiri, Al-Jarrah and Al-Wedyan (2003) developed an ANFIS system to find out the effect of machining variables and number of cutting flutes on the surface roughness of Alumic-79 in order to predict surface roughness and optimise cutting parameters. The inputs included in the model were spindle speed, feed rate and depth of cut, and two membership functions were chosen for each input. The surface roughness prediction was validated with an error of less than 5\%. A similar research work was previously reported by Al-Wedyan, Demirli and Bhat (2001), where special attention was paid to identify the best $n$-rule fuzzy models by a parametric search on the experimental data. The fuzzy model with the least number of rules with an acceptable error was reached with three membership functions for each input. Although it is not explicitly reported a validation procedure, it seems that the prediction model showed a similar error to Dweiri, Al-Jarrah and Al-Wedyan's (2003) research work. Lo (2003) applied an ANFIS system to surface roughness prediction in end milling operations paying special attention to the influence of different membership functions on the correct rate of surface roughness prediction. Both trapezoidal and triangular membership functions were studied, and the results reported a slightly higher correct rate of prediction when triangular functions were applied.

Other research works included sensor measurements in the ANFIS surface roughness model. Ho et al. (2002) developed an ANFIS model to predict surface roughness by computer vision in turning operations. The proposed ANFIS-based method outperformed a previous polynomial network-based method presented in Lee and Tarng (2001) in terms of modelling and prediction accuracy. The computer vision system, comprising a digital camera connected to a computer and the appropriate light sources, provided surface images that were analysed to calculate the arithmetic average of gray levels (number of shades of gray). This information as well as the cutting parameters were given, for a total of four inputs, to the ANFIS and the roughness value was then obtained. The performance of the ANFIS model showed a mean error of 4.6\%. Yang et al. (2006) proposed an adaptive surface roughness control system for end-milling operations based on cutting forces. The system was composed of two subsystems, one for predicting in-process surface roughness

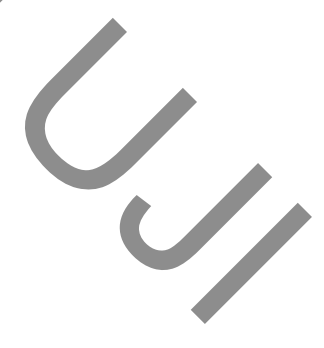


and another to control the feed rate that is adapted based on the predicted surface roughness. Fuzzy regions were defined for each parameter: cutting speed, feed rate, resulting force on the cutting plane, normal force to the cutting plane, surface roughness deviation and feed rate deviation.

In spite of the ability in extracting knowledge in form of rules from experimental data, any of the previous cited research works showed or analysed the learnt rules. Recently, Abellan-Nebot (2009) presented a cutting parameter optimisation procedure based on ANFIS models. For the surface roughness ANFIS model developed, the rules learnt during training were briefly described and analysed according to an expert machinist's opinion.

\section{Guidelines for neuro-fuzzy modelling}

The number of membership functions which define each variable can be defined by the expert/designer or can be extracted from experimental data. The number of membership functions per each cutting parameter input is usually chosen according to the experimental levels of each input. For example if the experimental data have three levels in the feed rate parameter, the fuzzy feed rate function should be composed of two membership functions (Dweiri et al., 2003). However, in case the input has a continuous value, it can be a priori difficult to identify the number of membership functions. In these cases, it is recommended the use of a subtractive clustering algorithm. This algorithm identify the best $n$-rule models with the least square error from the experimental data, so the number of membership functions to define these rules are explieitly generated. It is important to tune correctly the clustering parameters to identify the most important rules since a higher number of rules produces a higher number of membership functions and therefore, a higher number of experimental data is required for learning and validating the ANFIS model. An example of the application of this algorithm is shown by Al-Wedyan et al. (2001).

In general, the number of inputs and the number of membership functions per input have to be restricted to avoid an excessive number of parameters to be learnt. Excessive parameters require excessive experimental data which is unfeasible in most of industrial machining processes. The number of parameters to be learnt in an ANFIS model is defined by the expression:

$$
\text { Param }_{\text {free }}=\sum_{i=1}^{N}\left(M F S_{i} \times \text { Param }_{i}\right)+(N+1) \times N_{\text {rules }}
$$

where $N$ is the number of inputs, $M F S_{i}$ is the number of membership functions for the input $i, \operatorname{Param}_{i}$ is the number of parameters which define the membership function $i$, and $N_{\text {rules }}$ is the number of the total rules, which is the combination between the number of inputs and the number of membership functions per input. For example, three inputs and two membership functions per input generates $2^{3}$ rules. Therefore, according to Eq. (2D) an ANFIS model with three inputs and two membership functions per input, where each membership function is defined by two parameters, requires 44 parameters to be learnt. Obviously, the number of experimental data have to be considerably higher than the number of parameters to be learnt.

On the other hand, according to the results reported by Lo (2003), the adoption of different membership functions (triangular and trapezoidal) have a slight effect in the prediction model, but it seems not to be a relevant issue. In his experimentation, the ANFIS model for surface roughness achieved an accuracy of $96 \%$ with triangular membership functions whereas the accuracy was $93.3 \%$ with trapezoidal membership functions.

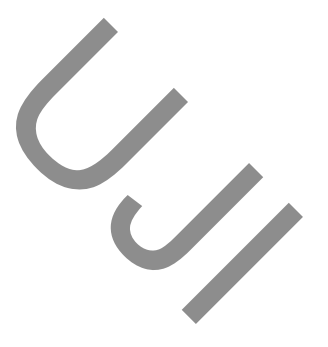




\subsection{Bayesian Networks}

\subsubsection{Introduction}

Process monitoring in machining requires the assimilation of numerous noisy and incomplete sources of evidence in order to infer the performance variables. Such inferences will necessarily be imperfect, and it would be valuable if the conclusions could be augmented with some quantifiable measure of its certainty or uncertainty. Unlike the previous Al techniques which are commonly used as universal function approximators, probabilistic inferencing techniques are better suited to deal with this kind of problems where uncertainty must be considered. Above all probabilistic inferencing techniques, Bayesian Networks (BNs) are probably the most widely applied technique. A BN provides a convenient formatism for representing conditional probabilistic relationships between attributes of interest, modelling and evaluating uncertainty (Dey and Stori, 2005).

$\mathrm{BN}$ are directed acyclic graphs in which each node represents a random variable that can take continuous or discrete values depending on the network topology. The nodes in the network are defined as parents and children whereas the arcs in the network signify the existence of direct causal influences between the linked variables. If there is a causal link from node $A$ to $B$, it is said that $B$ is child of $A$ and $B$ is a parent of $A$. For each variable $A$ with parents $\mathrm{B}_{1}, \ldots, \mathrm{B}_{n}$ is attached a conditional probability table $\mathrm{P}\left(\mathrm{A} \mid \mathrm{B}_{1}, \ldots, \mathrm{B}_{n}\right)$ which define the strengths of these causal links. The directionality of the arrows is essential for displaying causality and non-transitive dependencies. The causal relationships expressed through the network structure and the conditional probability tables are based on prior knowledge, experience, or statistically observed correlations learnt from experimental data (Dey and Stori, 2005). The BN performance is based on propagation probabilities when it is known a specific variable state, called evidence. Therefore, $\mathrm{BN}$ shows the states of each node and its associated probability according to the evidences in the network.

Two mainly types of reasoning can be conducted by BNs for part accuracy prediction: predictive reasoning and diagnostic reasoning. The first reasoning method lets estimate the probability of each part accuracy range given certain manufacturing requirements such as cutting speed, depth of cut, etc. The second reasoning method lets estimate which are the probabilities of unobserved variables if the part accuracy is known(Correa et al., 2008a).

For the sake of understanding BNs, two BN examples reported in Correaetal.'s (2008a) and Abellan-Nebot et al.'s (2006a) research work are shown in Fig. 7 . The networks relate surface roughness ranges with cutting parameters such as feed rate (fz), cutting speed (V/c), spindle speed (rpm) and sensor measurements such as forces (FT), vibrations and spindle load. Both networks are used to predict surface roughness given different evidences. The first figure shows the probability of machining parts within a smooth, fine, semi-fine and medium surface roughness quality given the feed rate, the spindle speed and the cutting forces measurement. The BN network predicts a probability of $0.76,0.01,0.20$ and 0.01 for these surface roughness ranges respectively. The second figure shows a similar procedure where both surface roughness ranges and cutting tool flank wear are predicted. According to the evidence propagation, the $\mathrm{BN}$ network predicts a surface roughness range of 3.3-4.8 $\mu m$ with a probability of $75 \%$ whereas there is a probability of $25 \%$ to obtain a surface roughness higher than $4.8 \mu \mathrm{m}$.

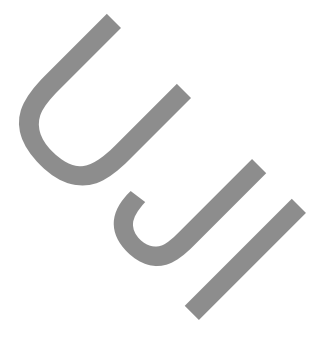




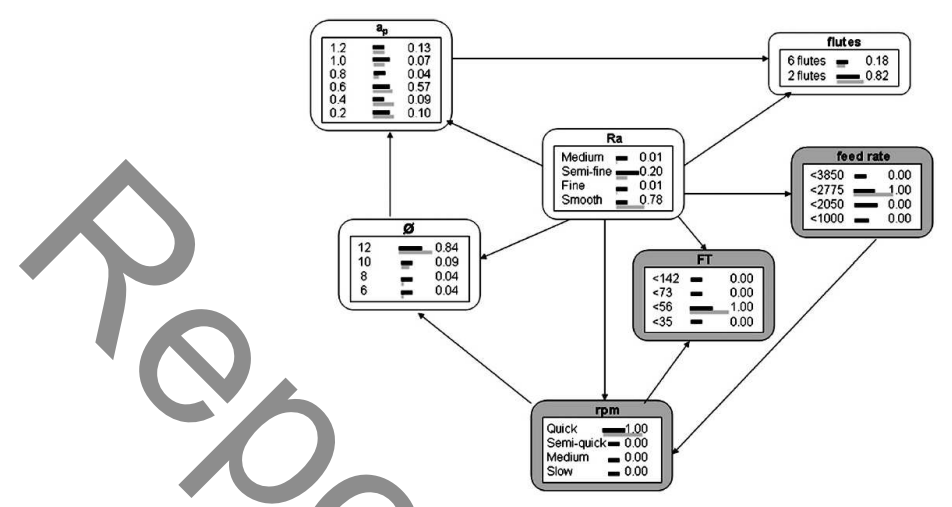

(a)

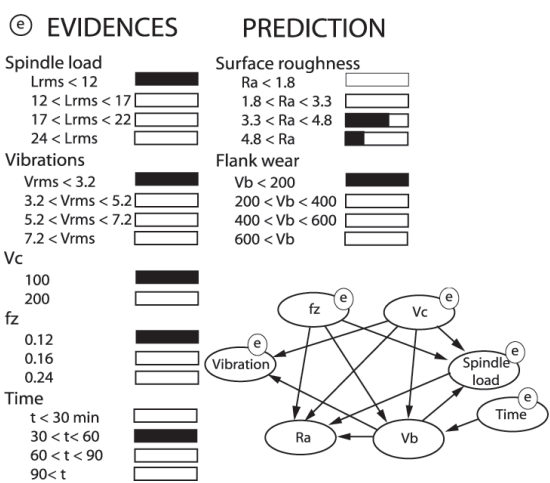

(b)

Figure 7 Examples of BNs models applied to surface roughness prediction. Correa et al (2008a) presents in Fig. (a) how the surface roughness is inferred knowing as evidences the feed rate, the forces (FT) and the spindle speed (rpm). The BN predicts a smooth surface roughness with a probability of 76\%. Abellan-Nebot et al (2006a) presents in Fig. (b) how the surface roughness predicts a range of $3.3-4.8 \mu \mathrm{m}$ with a probability of $75 \%$ knowing the process variables cutting speed ( $\mathrm{Vc}$ ), feed rate (fz), vibration value, machining time and spindle load.

\subsubsection{Bayesian Models applied to part accuracy prediction}

In spite of the potential use of BNs, their application in machining have been mainly focused on fault cause diagnosis and tool wear prediction (Dey and Stori, 2005). Recently, some authors have extended their application to surface roughness prediction since surface roughness tends to show an important stochastic behaviour. Abellan-Nebot et al. (2006a) applied BNs for cutting-tool wear diagnosis and surface roughness ranges prediction in milling operations. They compared three different $\mathrm{BN}$ models according to different discretisation ranges since variables in $\mathrm{BN}$ have to be defined in discrete ranges. The variables included in the model were: spindle speed, feed per tooth, root-mean square vibrations, root-mean square spindle load, operating time, surface roughness and flank wear. Three BN models were developed: model-1 for low discretisation ( 5 discretisated intervals per variable); model-2 for medium discretisation (7 intervals) and model-3 for high discretisation (10 intervals). Due to the limited experimental data (134 data records), it was needed to provide a prior process knowledge during the training step. Fundamental physical relationships were included by adding well-known causal relationships in the BN structure. The remaining causal relationships were learnt from experimental data by the learning algorithm Necessary Path Condition (NPC). The results showed the importance of the level of discretisation. Models with high discretisation tended to be more precise but less reliable whereas the model with low discretisation presented low precision. The authors concluded that a right trade-off between discretisation and reliability could generate effective $\mathrm{BN}$ models for prediction in this domain. However, they remarked that a higher discretisation requires higher experimental data to learn the conditional probabilistic relationships which is an important limitation in industrial machining systems.

Correa et al. (2008a) presented BN models for surface roughness prediction where the discretisation algorithms K-means and Fuzzy K-means were applied. They compared two BN models to predict the surface roughness ranges of machined parts. The first model 
was based on Nave Bayes structure whereas the second model was based on the TreeAugmented Nave Bayes (TAN) structure. A data set of 250 records was used for training and validating both models. The results reported that the TAN structure was able to classify the surface roughness with an accuracy of $81.2 \%$, slightly higher than the Nave Bayes structure which achieved an accuracy of $76 \%$. Although the previous research works do not deal directly with cutting parameters selection for process planning, both studies remarked the potential use of BNs to recommend the cutting parameters that yield, with certain probability, a desired surface roughness level.

As a novel approach applied to part accuracy prediction, some authors have recently compared the performance of BNs with other common approaches such as ANNs or statistical regressions. Correa, Bielza and Pamies-Teixeira (2008b) compared a MLP ANN with a topology 7-11-4 versus a BN model with a TAN structure for quality detection in high speed machining processes. The results reported a better performance of the BN classifier than the ANN classifier, with an accuracy of $96.3 \%$ and $94.8 \%$ respectively. Two statistical test were condueted confirming that the BN accuracy was statistically superior to ANN accuracy. The use of $\mathrm{BN}$ was also preferred since this $\mathrm{AI}$ technique was easier to interpret than ANNs. Abellan-Nebot et al. (2006b) also compared the performance of BN versus ANN models. The results showed that the BN model presented similar precision for surface roughness prediction than the ANN model. However, BN predictions showed $33.7 \%$ less uncertainty than ANN predictions whereas the ANN predictions were more reliable. The lack of reliability of BN was assumed to be due to the high number of experimental data required in $\mathrm{BN}$ models.

\subsubsection{Guidelines for Bayesian Modelling}

Predictions with BN are not very aceurate, and their accuracy depends on the variable discretisation ranges. A higher discretisation can lead to a higher predictions accuracy, however, the experimental data required for modelling increase rapidly with discretisation. As experimental data in machining is always an important limitation, low discretisation variables are recommended in order to use enough data to extract the associated probabilities between cutting and process variables. For discretisation purposes, it is also recommended the use of discretisation algorithms such as K-means and Fuzzy K-means as it was reported by Correa, Bielza, de Ramirez and Alique (2008a).

The low accuracy in BN predictions difficulties the use of these models in surface roughness or dimensional deviation predictions. However, BN can be yery useful to identify patterns, so applications such as diagnosis of surface roughness ranges or dimensional deviation ranges can be successfully implemented in order to predict if a machined part is within or outside customer specifications with a certain level of uncertainty.

On the other hand, BN requires a high number of experimental data to learn the model structure and the conditional probability tables. In order to reduce the experimental data, previous expert knowledge about the machining process can be added into the network through predefined causal relationships. Therefore, the learning process can be improved adding some well-known machining relationships such as: feed rate causes surface roughness, feed rate causes an increase of spindle load, no causal relationship between cutting parameters (cutting speed, feed rate), etc. (see Abellan-Nebot et al.'s (2006a) research work). In addition, algorithms to learn BN structure should be properly chosen to deal with a low number of experimental data. Accordingly, NPC algorithm which is an extension of the PC algorithm is recommended for part accuracy prediction in Abellan-Nebot et al.'s (2006a) research work. Through this algorithm, the designer can add his experience fixing

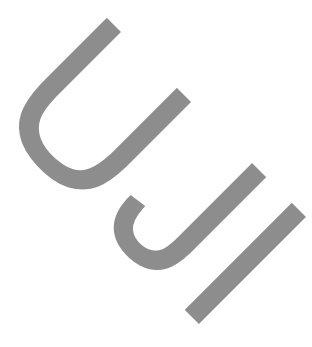


causal arcs during the learning process. Causal relationships can be also acquired by an ANOVA analysis as it is reported by Dey and Stori (2005).

\section{Guidelines for AI techniques selection}

The AI approaches reviewed above have been applied for both surface roughness and dimensional deviation prediction in machining. However, there are special considerations that should be taken into account in order to select the most appropriate AI technique for a given purpose (refer to Fig. 8). According to the literature review, the main advantages and drawbacks for each AI technique and its recommended application field are defined as follows

- Artificial Neural Networks (ANN): Applications where there is no purpose of knowledge extraction, and there is no previous knowledge of the process (or if there is previous knowledge, this knowledge is not intended to be added into the model). Applications where a high accuracy prediction is required. Applications where there is no extrapolation and a good generalisation is required. Applications where the experimental data set is composed of a medium number of samples. Applications where only the prediction or diagnosis is required, and the inverse problem such as cutting parameters evaluation to assure a specific output value is not considered. In general, ANNs are the main tool forboth surface roughness and dimensional deviation prediction.

- Fuzzy inference systems: Applications where there is enough knowledge from the process and this knowledge is intended to be added into the model. Applications where the understanding of the process prevails over the model accuracy. Applications where extrapolation can occur and the gemeral process behaviour is expected to be smooth. Applications where the experimentaldataset is composed of low/medium number of samples since part of the model is deyeloped using previous knowledge. Applications where a part from a specific variable prediction, the inverse problem has to be solved. In general, fuzzy inference systems are used for surface roughness prediction and cutting parameter selection given a surface roughness specification.

- Adaptive Neuro-fuzzy Inference Systems (ANFIS): Applications where it is desired to add previous knowledge and/or to extract hidden knowledge from experimental data in a rule-form. Applications where extrapolation and generalisation ability are demanded. Applications with a moderate accuracy requirement. Applications where the experimental data set is composed of a medium number of samples. Applications where a part from a specific variable prediction, the inverse problem has to be solved. Since ANFIS are a hybridisation of ANN and fuzzy systems, the recommended applications are similar to both ANN and fuzzy applications.

- Bayesian Networks (BN): Applications where it is desired to add previous knowledge and/or to extract hidden knowledge in a form of causal relationships and probabilities. Applications where low accuracy prediction is required but with high reliability. Applications which have an important stochastic component. Applications where the experimental data set is composed of large/very large number of samples depending on the variable discretisation ranges and the expected accuracy. Applications where partial observations are required, i.e. not all the variables of the model are 


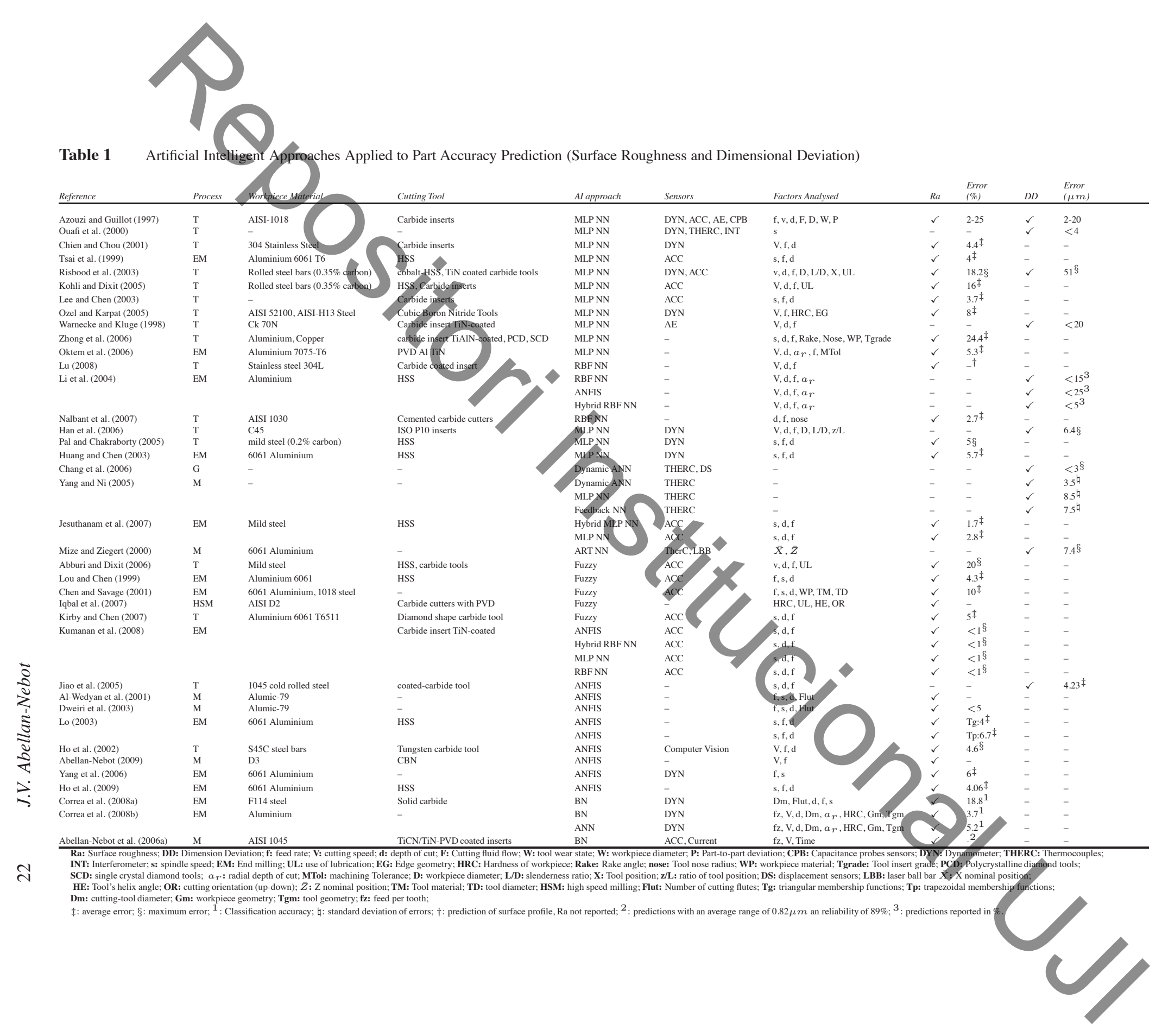


available to estimate the output variable. $\mathrm{BN}$ is recommended for surface roughness ranges prediction in order to detect if the machined parts are within or outside specifications. BNs are also recommended for cutting parameter selection to meet part specifications.

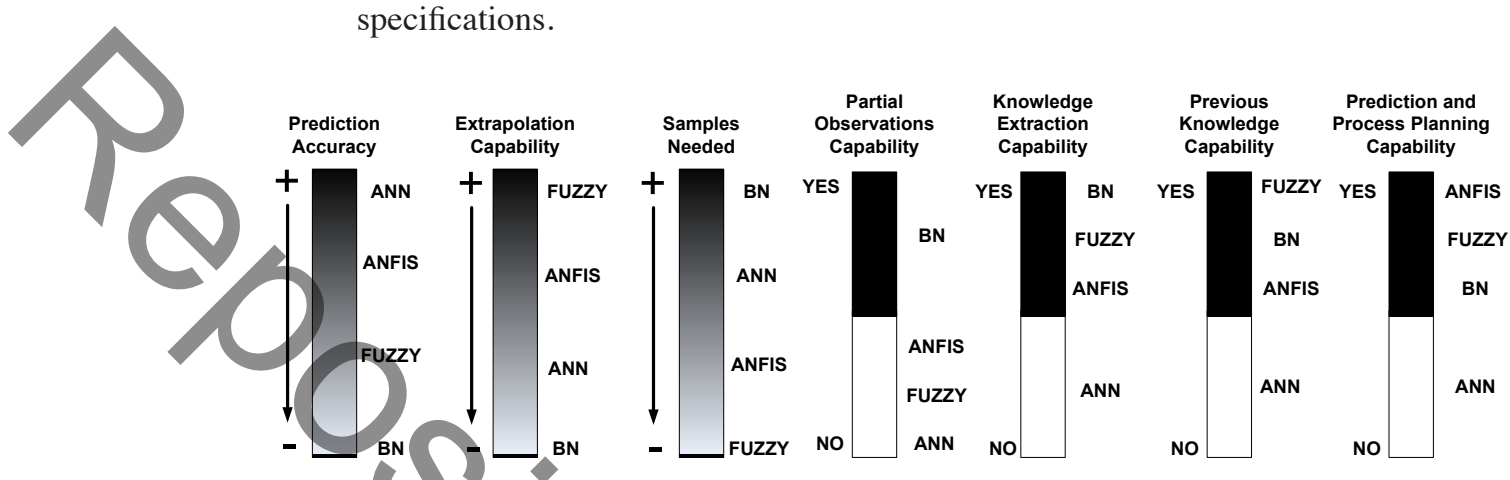

Figure 8 Basic guidelines for AI selection according to model requirements.

\section{Conclusions}

Artificial Intelligent (AI) approaches have been intensively applied in machining processes in order to increase part accuracy. In spite of the large volume of academic research work in this field, there is no review on AI applied to part accuracy prediction and it is difficult to find a summary of these AI techniques in order to facilitate their use. This paper has tried to overcome both limitations summarising the main AI approaches applied to part accuracy prediction namely Artificial Neural Networks, Fuzzy systems, Adaptive-Neuro Fuzzy Inference Systems, and Bayesian Networks, and the main research works reported in the literature. In addition, useful guidelines for applying these AI techniques in machining were provided according to previous research recommendations. To facilitate the use of AI techniques in intelligent machining, it was also presented a guideline for AI selection, considering the main advantages and drawbacks of each AI reviewed. This paper can be considered a brief guide for all practitioners interested in applying artificial intelligence techniques in machining processes for part quality improvement.

\section{Acknowledgements}

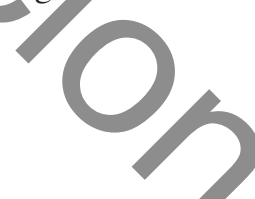

This work has been partially supported by Fundació Caixa-Castelló Bancaixa (Research Promotion 2007). The author would like to acknowledge the many helpful suggestions of the anonymous reviewers.

\section{References}

Abburi, N. R. and Dixit, U. S. (2006) 'A knowledge-based system for the prediction of surface roughness in turning process', Robotics and Computer-Integrated Manufacturing, Vol. 22, No. 4, pp. 363-372.

Abellan-Nebot, J. V. (2009) 'Optimization of cutting parameters in milling: expert machinist knowledge versus soft computing methods', International Journal of Mechatronics and Manufacturing Systems, Vol. In Press. 
Abellan-Nebot, J. V., Morales-Menendez, R., Vallejo, A. J. and Rodriguez, C. (2006a) 'Surface and cutting toolwear diagnosis based on bayesian networks', In 6th IFAC Safe Process.

Abellan-Nebot, J. V., Morales-Menendez, R., Vallejo, A. J. and Rodriguez, C. (2006b) 'Comparison of modelling approaches in surface roughness and cutting tool-wear condition for face milling operations', CIRP-2nd International Conference. High Performance Cutting. Vancouver, Canada.

Abellan-Nebot, J. V., Romero, F., Morales-Menendez, R., Siller, H. and Vila, C. (2008) 'Adaptation of surface roughness models based on ai in changing environments', Proc. of the special session of the 7th Mexican International Conference on Artificial Intelligence 2008, MICAI-08. IEEE Computer Society. Atizapan de Zaragoza, Mexico, pp. 370-376.

Al-Wedyan, H., Demirli, K. and Bhat, R. (2001) 'A technique for fuzzy logic modeling of machining process', IFSA World Congress and 20th NAFIPS International Conference, 2001.Joint 9th, pp. 3021-3026.

Azouzi, R. and Guillot, M. (1997) 'On-line prediction of surface finish and dimensional deviation in turning using neural network based sensor fusion', International Journal of Machine Tools and Manufacture, Vol. 37, No. 9, pp. 1201-1217.

Benardos, P. G. and Vosniakos, G. C. (2002) 'Prediction of surface roughness in cnc face milling using neural networks and taguchi's design of experiments', Robotics and Computer-Integrated Manufacturing, Vol. 18, No. 5-6, pp $343-354$.

Benardos, P. G. and Vosniakos, G. C. (2003) 'Predicting surface roughness in machining: a review', Int. Journal of Machine Tools and Manufacture, Vol. 43, No. 8, pp. 833-844.

Bernauer, E. and Demmou, H. (1993) 'Temporal sequence learning with neural networks for process fault detection', Systems, Man and Cybernetics, 1993.'Systems Engineering in the Service of Humans', Conference Proceedings, pp. 375-380.

Chang, C. W., Kang, Y., Chen, Y. W., Cha, M. H. and Wang, Y. P. (2006) 'Thermal deformation prediction in machine tools by using neural network', Neural Information Processing, Pt 2, Proceedings, Vol. 4233, pp. 850-859.

Chen, G. (2000) Rapid Volumetric Error Mapping and Compensation for a Three-axis Machining Center, Thesis/dissertation.

Chen, J. C. and Savage, M. (2001) 'A fuzzy-net-based multilevel in-process surface roughness recognition system in milling operations', International Journal of Adyanced Manufacturing Technology, Vol. 17, No. 9, pp. 670676.

Chien, W. T. and Chou, C. Y. (2001) 'The predictive model for machinability of 304 stainless steel', Journal of Materials Processing Technology, Vol. 118, No. 1-3,pp. 442-447,

Colak, O., Kurbanoglu, C. and Kayacan, M. C. (2007) 'Milling surface roughness prediction using evolutionary programming methods', Materials and Design, Vol. 28, No 2, pp 657-666.

Correa, M., Bielza, C. and Pamies-Teixeira, J. (2008b) 'Comparison of bayesian networks and artificial neural networks for quality detection in a machining process', Expert Systems with Applications, Vol. In Press.

Correa, M., Bielza, C., de Ramirez, M. and Alique, J. (2008a) 'A bayesian network model for surface roughness prediction in the machining process', International Journal of Systems Science, Vol. 39, No. 12, pp. 1181-1192.

Demuth, H., Beale, M. and Hagan, M. (2008) Neural Network Toolbox: User's Guide, The Math Works Inc.

Dey, S. and Stori, J. A. (2005) 'A bayesian network approach to root cause diagnosis of process variations', Int. J. of Machine Tools and Manufacture, Vol. 45, No. 1, pp. 75-91.

DIN4760 (1982) 'Form deviations; concepts; classification system.', Deutches Institut Fuer Normung, e.V.

Dixit, U. S. and Chandra, S. (2003) 'A neural network based methodology for the prediction of roll force and roll torque in fuzzy form for cold flat rolling process', Int. J. of Adv. Manufacturing Technology, Vol. 22, No.11-12, pp. 883-889.

Dweiri, F., Al-Jarrah, M. and Al-Wedyan, H. (2003) 'Fuzzy surface roughness modeling of cnc down milling of alumic-79', Journal of Materials Processing Technology, Vol. 133, No. 3, pp. 266-275.

Eberhart, R. C., Simpson, P. and Dobbins, R. (1996) Computational intelligence PC tools, New York: In: AP professional.

Freeman, J. A. and Skapura, D. M. (1991) Neural Networks: Algorithms, applications, and programming techniques, Addison-Wesley Publishing Company, Inc.

Grzesik, W. (1996) 'A revised model for predicting surface roughness in turning', Wear, Vol. 194, No. 1-2, pp. 143-148.

Han, R., Cui, B. and Guo, J. (2006) 'In-process monitoring of dimensional errors in turning slender bar using artificial neural networks', 'CSCWD (Selected Papers)', pp. 277-286.

Haykins, S. (1994) Neural Networks: A Comprehensive Foundation, New Jersey. 
Ho, S. Y., Lee, K. C., Chen, S. S. and Ho, S. J. (2002) 'Accurate modeling and prediction of surface roughness by computer vision in turning operations using an adaptive neuro-fuzzy inference system', Int. J. of Machine Tools and Manuf., Vol. 42, No. 13, pp. 1441-1446.

Ho, W., Tsai, J., Lin, B. and Chou, J. (2009) 'Adaptive network-based fuzzy inference system for prediction of surface roughness in end milling process using hybrid taguchi-genetic learning algorithm', Expert Systems with Applications, Vol. 36, No. 2, Part 2, pp. 3216 - 3222.

Huang, B. and Chen, J. C. (2003) 'An in-process neural network-based surface roughness prediction (inn-srp) system using a dynamometer in end milling operations', International Journal of Advanced Manufacturing Technology, Vol. 21, No. 5, pp. 339-347.

Iqbal, A., He, N., Li, L. and Dar, N. U. (2007) 'A fuzzy expert system for optimizing parameters and predicting performance measures in hard-milling process', Expert Systems with Applications, Vol. 32, No. 4, pp. 10201027.

Ishibuchi, H. and Tanaka, H. (1991) 'Regression analysis with interval model by neural networks', Proc of the IEEE Int Joint Conf on Neural Networks, pp. 1594-1599.

Ivester, R. W., Kennedy, M., Davies, M., Thiele, R. S. J., Furness, R. and Athavale, S. (2000) 'Assessment of machining models: Progress report', Machining Science and Technology, Vol. 4, No. 3, pp. 511-538.

Jain, A. K., Mao, J. C. and Mohiuddin, K. M. (1996) 'Artificial neural networks: A tutorial', Computer, Vol. 29, No. 3, pp. 31-44.

Jain, L. C. and Martin, N. M. (1998) Fusion of Neural Networks, Fuzzy Systems and Genetic Algorithms: Industrial Applications

Jesuthanam, C. P., Kumanan, S. and Asokan, P. (2007) 'Surface roughness prediction using hybrid neural networks', Machining Science and Tech., Vol. 11, No. 2, pp. 271-286.

Jiao, Y., Pei, Z. J., Lei, S., Lee, E. S.and Fisher, G. R. (2005) 'Fuzzy adaptive networks in machining process modelling: dimensional error prediction for turning operations', International Journal of Production Research, Vol. 43, No. 14, pp. 2931-2948.

Kasabov, N. K. (1996) Foundations of Neural Netyorks, Fuzzy Systems, and Knowledge Engineering.

Kirby, E. D. and Chen, J. C. (2007) 'Development of a fuzzy-nets-based surface roughness prediction system in turning operations', Computers and Industrial Engineering, Vol. 53, No. 1, pp. 30-42.

Kohli, A. and Dixit, U. S. (2005) 'A neural-network-based methodology for the prediction of surface roughness in a turning process', International Journal of Advanced Manufacturing Technology, Vol. 25, No. 1-2, pp. $118-129$.

Kumanan, S., Jesuthanam, C. P. and Kumar, R. A. (2008) 'Application of multiple regression and adaptive neuro fuzzy inference system for the prediction of surface roughness', Int Journal of Advanced Manufacturing Technology, Vol. 35, No. 7-8, pp. 778-788.

de Lacalle, L., A.Lamikiz, J.A.Sanchez and M.A.Salgado (2004) 'Effects of tool deflection in the high-speed milling of inclined surfaces', International Journal of Advanced Manufacturing Technology, Vol. 24, pp. 621631.

Lee, B. Y. and Tarng, Y. S. (2001) 'Surface roughness inspection by computer vision in turning operations', International Journal of Machine Tools and Manufacture, Vol. 41, No. 9, pp.1251-1263.

Lee, S. S. and Chen, J. C. (2003) 'On-line surface roughness recognition system using artificial neural networks system in turning operations', International Journal of Advanced Manufacturing Technology, Vol. 22, No. 7-8, pp. 498-509.

Li, X. L., Guan, X. P. and Li, Y. (2004) 'A hybrid radial basis function neural network for dimensional error prediction in end milling', Advances in Neural Networks - Isnn 2004, Pt 2, Vol. 3174, pp. 743-748.

Lo, S. P. (2003) 'An adaptive-network based fuzzy inference system for prediction of workpiece surface roughness in end milling', Journal of Materials Processing Technology, Vol. 142, No. 3, pp. 665-675.

Lou, S. J. and Chen, J. C. (1999) 'In-process surface roughness recognition (isrr) system in end-milling operations', International Journal of Advanced Manufacturing Technology, Vol. 15, No. 3, pp. 200-209.

Lu, C. (2008) 'Study on prediction of surface quality in machining process', Journal of Materials Processing Technology, Vol. 205, No. 1-3, pp. 439-450.

van Luttervelt, C. A., Childs, T. H. C., Jawahir, I. S., Klake, F. and Venuvinod, P. K. (1998) 'Present situation and future trends in modeling of machining operations - report of the cirp working group on modeling of machining operations', Annals CIRP, Vol. 47, No. 2.

van Luttervelt, C. A. and Peng, J. (1999) 'Symbiosis of modelling and sensing to improve the accuracy of workpieces in small batch machining operations', Int. J. of Adv. Manufacturing Technology, Vol. 15, No. 10, pp. 699-710. 
Mize, C. D. and Ziegert, J. C. (2000) 'Neural network thermal error compensation of a machining center', Precision Engineering-Journal of the International Societies for Precision Engineering and Nanotechnology, Vol. 24, No. 4, pp. 338-346.

Nalbant, M., Gokkaya, H. and Toktas, I. (2007) 'Comparison of regression and artificial neural network models for surface roughness prediction with the cutting parameters in cnc turning', Model. Simul. Eng., Vol. 2007, No. 2, pp. 1-14.

Oktem, H., Erzurumlu, T. and Erzincanli, F. (2006) 'Prediction of minimum surface roughness in end milling mold parts using neural network and genetic algorithm', Materials and Design, Vol. 27, No. 9, pp. 735-744.

Ouafi, A. E., Guillot, M. and Bedrouni, A. (2000) 'Accuracy enhancement of multi-axis cnc machines through on-line neurocompensation', Journal of Intelligent Manufacturing, Vol. 11, No. 6, pp. 535-545.

Ozel, T. and Karpat, Y. (2005) 'Predictive modeling of surface roughness and tool wear in hard turning using regression and neural networks', International Journal of Machine Tools and Manufacture, Vol. 45, No. 4-5, pp. $467-479$.

aal, S. K. and Chakraborty, D. (2005) 'Surface roughness prediction in turning using artificial neural network', Neural Computing and Applications, Vol. 14, No. 4, pp. 319-324.

Raghu, A and Melkote, S. N. (2005) 'Modeling of workpiece location error due to fixture geometric error and fixture-workpiece compliance', Journal of Manufacturing Science and Engineering-Transactions of the Asme, Vol. 127, No. 1, pp. 75-83.

Raksiri, C. and Parnichkun, M. (2004) 'Geometric and force errors compensation in a 3-axis cnc milling machine', International Journal of Machine Tools and Manufacture, Vol. 44, No. 12-13, pp. 1283-1291.

Ramesh, R., Mannan, M. A. and Poo, A. N. (2000) 'Error compensation in machine tools - a review part i: geometric, cutting-force induced and fixture-dependent errors', Int. J. of Machine Tools and Manufac., Vol. 40, No. 9, pp. 1235-1256.

Risbood, K. A., Dixit, U. S. and Sahasrabudhe, A. D. (2003) 'Prediction of surface roughness and dimensional deviation by measuring cutting forces and vibrations in turning process', J. of Materials Proc. Tech., Vol. 132, No. 1-3, pp. 203-214.

Sonar, D. K., Dixit, U. S. and Ojha, D. K. (2006) 'The application of a radial basis function neural network for predicting the surface roughness in a turning process', International Journal of Advanced Manufacturing Technology, Vol. 27, No. 7-8, pp. 661-666.

Stephenson, D. A. and Agapiou, J. S. (1997) Metal cutting theory and practice, New York: Marcel Dekker, Inc.

Tsai, Y. H., Chen, J. C. and Lou, S. J. (1999) 'An in-process surface recognition system based on neural networks in end milling cutting operations', International Journal of Machine Tools and Manufacture, Vol. 39, No. 4, pp. 583-605.

Warnecke, G. and Kluge, R. (1998) 'Control of tolerances in turning by predictive control with neural networks', Journal of Intelligent Manufacturing, Vol. 9, No. 4, pp. 281-287.

Yang, H. and Ni, J. (2005) 'Dynamic neural network modeling for nonlinear, nonstationary machine tool thermally induced error', International Journal of Machine Tools and Manufacture, Vol. 45, No. 4-5, pp. 455-465.

Yang, L. D., Chen, J. C., Chow, H. M. and Lin, C. T. (2006) 'Fuzzy-nets-based in-process sufface roughness adaptive control system in end-milling operations', International Journal of Advanced Mañfacturing Technology, Vol. 28, No. 3-4, pp. 236-248.

Zhong, Z. W., Khoo, L. P. and Han, S. T. (2006) 'Prediction of surface roughness of turned surfaces using neural networks', International Journal of Advanced Manufacturing Technology, Vol. 28, No. 7-8, pp. 688-693.

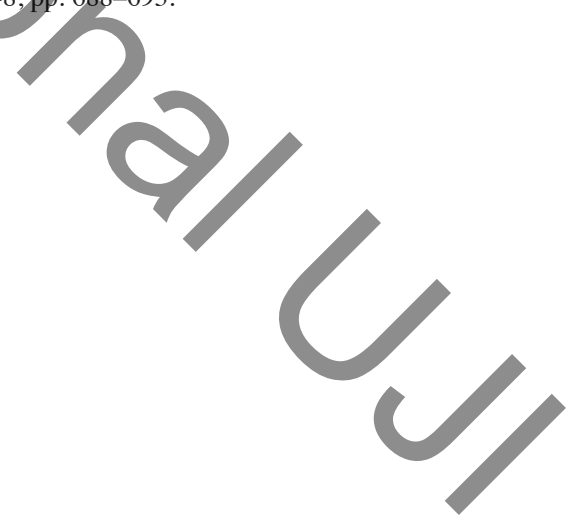

